\title{
Highly controllable and reliable ultra-thin Parylene deposition
}

\author{
Yaoping Liu', Dongyang Kang ${ }^{2}$, Wangzhi Dai ${ }^{1}$, Haida Li ${ }^{1}$, Wei Wang ${ }^{1,2,3^{*}}$ (D) and Yu-Chong Tai ${ }^{2 *}$
}

\begin{abstract}
Thanks to the excellent barrier property and fabrication accessibility, Parylene has been actively used in the microelectromechanical system. An ultra-thin Parylene film with thickness smaller than $100 \mathrm{~nm}$ is usually required to precisely tune the surface property of substrate or protect the functional unit. The commercially available regular Parylene deposition is a dimer mass determined chemical vapor deposition process with a high output (i.e. a low deposition precision in term of thickness control), around $1.6 \mu \mathrm{m} / \mathrm{g}$ (the ratio of film thickness to the loaded dimer mass) for the machine in the author's lab. Therefore, it is hard to controllably and reliably prepare a Parylene film with thickness smaller than $100 \mathrm{~nm}$, which requires a dimer mass less than $62.5 \mathrm{mg}$. This paper reported a method to prepare ultrathin Parylene films with the nominal thickness down to $1 \mathrm{~nm}$. A home-made deposition chamber was put inside and connected with the regular machine chamber through a microfabricated orifice with feature size smaller than $1 \mathrm{~mm}$. According to the free molecular flow theory, the pressure inside the deposition chamber can be predictably and controllably reduced, thereby an ultra-low output of Parylene deposition, as low as $0.08 \mathrm{~nm} / \mathrm{g}$, was successfully obtained. The deposition precision was increased by 4 orders of magnitude compared to that of a direct Parylene deposition. This highly controllable and reliable ultra-thin Parylene deposition technique will find promising applications in flexible electronics and biomedical microdevices.
\end{abstract}

Keywords: Ultra thin Parylene deposition, Free molecular flow, Orifice

\section{Background}

As one of the most widely-used polymers in the microelectromechanical system (MEMS), Parylene played active roles in flexible electronics and biomedical microdevices, such as substrates for flexible microelectrode array [1-3], protection layer for microdevices [4-9], etc. Generally, the Parylene deposition process consists of three steps: (1) Parylene dimers were vaporized in the sublimation furnace at temperature of $150-175{ }^{\circ} \mathrm{C}$. (2) The vaporized dimers were then pyrolyzed into monomers in the hightemperature $\left(\sim 690^{\circ} \mathrm{C}\right)$ furnace. (3) The monomers then entered the machine chamber and deposited onto the exposed substrates at room temperature. The thickness of the deposited film is determined by the mass of loaded dimer. The experiments conducted on the PDS coating

\footnotetext{
*Correspondence: w.wang@pku.edu.cn; yctai@mems.caltech.edu 1 Institute of Microelectronics, Peking University, Beijing 100871, China

2 Electrical Engineering, Caltech, Pasadena, CA 91125, USA

Full list of author information is available at the end of the article
}

system 2010 (SCS) in the author's lab showed that the ratio of the deposited film thickness to the loaded dimer mass (the deposition output) is around $1.6 \mu \mathrm{m} / \mathrm{g}$, which guarantees an efficient Parylene coating for regular applications.

Recently, ultra-thin Parylene films with the thickness less than $100 \mathrm{~nm}$ ( $62.5 \mathrm{mg}$ dimer according to the aforementioned output), has been studied for various interesting applications $[4,5,7,8,10]$. Nishinaka et al. [7] utilized an ultra-thin Parylene coating ( $25 \mathrm{~nm}-38 \mathrm{~nm}$ ) to stabilize the electrode/electrolyte interface while keeping the electrode conductive. Lin et al. [10] reported that a $40 \mathrm{~nm}$ thick Parylene film had a switching resistor character, which is capable of preparing novel memristors. Meanwhile, by depositing an ultra-thin Parylene film with thickness of $640 \mathrm{~nm}$ onto polydimethylsiloxane (PDMS) substrates, Kang et al. [11] reported the water vapor transmission rate of PDMS can be precisely tuned. However, reducing the loaded dimer mass to deposit a thin Parylene film is not well controllable and 
reliable, especially aiming at an ultra-small thickness. For example, for the $10 \mathrm{~nm}$ thick Parylene deposition, only one dimer granule, or even less, will be required and a $0.6 \mathrm{mg}$ mass variation could lead to a $10 \%$ deviation from the target thickness. On the other hand, the ultra-small dimer mass made the deposition process extremely short and unstable. Compared to a long-term and stable pressure in the direct Parylene deposition with a large target thickness, the pressure inside the machine chamber (i.e. base pressure pluses monomer pressure $\left.\left(P_{M}\right)\right)$ in a short deposition always failed to reach its set value $\left(P_{S}\right)$ before the deposition completes, as shown in Fig. 1. Therefore, a highly controllable and reliable ultra-thin Parylene deposition is still lack and requires further study.

Effusion is a process when gas molecules go through an orifice whose size is smaller than the mean free path of the gas. When the ratio of the mean free path of gas to the orifice feature size, known as the Knudsen Number $(\mathrm{Kn})$, is greater than 1 , the gas flow can be described as the free molecular flow. Under this circumstance, the collations between molecules could be neglected and the gas molecules would go through the orifice at a certain possibility. As a consequence, the gas effusion through a precise orifice leads to a controllable and reliable pressure drop in between of the two sides of the orifice. The effusion process was utilized to allow a small fraction of Parylene monomers inside the machine chamber to go through an orifice and enter an additional deposition chamber. This effusion process enabled a huge pressure difference between the two sides of the orifice. As the deposition rate of Parylene was reported to be proportional to the deposition pressure at a certain temperature [12], this pressure difference could lead to a difference in

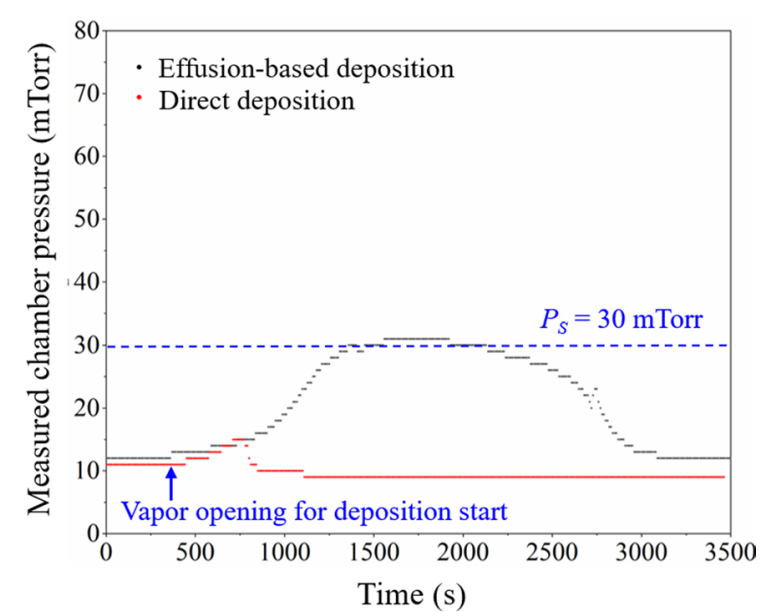

Fig. 1 Variation of the recorded machine chamber pressure along with time in the present effusion-based deposition and direct deposition processes. In the depositions, the target thicknesses were both $30 \mathrm{~nm}$ deposition rate, which thereby realize a long and stable deposition of ultra-thin Parylene films. Previous works have already demonstrated this concept, where $<500 \mathrm{~nm}$ Parylene coatings were successfully obtained $[6,9]$.

In this work, we further explored the capability of the ultra-thin Parylene $\mathrm{C}$ deposition by using a well-prepared micro orifice to achieve a highly controllable and reliable effusion-based process. A free molecule flow model was also established to describe this effusion-based deposition process.

\section{Method \\ Setup of the effusion-based deposition system}

The schematic illustration of the deposition system is shown in Fig. 2a. The wafers were placed inside the home-made deposition chambers (for example, D1-D3 showed in Fig. 2a), which were put inside the regular machine chamber. On the cap of each deposition chamber, there was a silicon chip with a microfabricated orifice. The silicon chip was taped on the cap, making the orifice the only connection between the regular machine chamber and the home-made deposition chamber. As the mean free path of the Parylene monomers inside the machine chamber is $0.81 \mathrm{~mm}\left(T=294 \mathrm{~K}, P_{M}=22 \mathrm{mTorr}\right)$, the size of the orifice ranging from tens to hundreds micrometers could enable a molecular effusion of monomers. As the effusion rate is proportional to the area of the orifice, Parylene films with different thicknesses could be obtained in a single effusion-based deposition run by using deposition chambers with different orifice sizes. For all the depositions in the present work, the loaded dimer mass was $10 \mathrm{~g}$ without specific notation.

\section{Fabrication of the orifice chip}

Figure $2 b$ schematically described the utilized fabrication process of the orifice chip. First, $300 \mathrm{~nm} \mathrm{SiO}{ }_{2}$ and $100 \mathrm{~nm}$ $\mathrm{Si}_{3} \mathrm{~N}_{4}$ layers were deposited onto a silicon wafer through low pressure CVD (LPCVD), severed as a mask for the wet etching of Silicon. Then the orifice was defined by photolithography and dry etching of the $\mathrm{Si}_{3} \mathrm{~N}_{4}$ and $\mathrm{SiO}_{2}$ layers by the reaction ion etching (RIE). Finally, $\mathrm{KOH}$ was used to etch silicon until through and then the $\mathrm{Si}_{3} \mathrm{~N}_{4}$ and $\mathrm{SiO}_{2}$ layers were stripped off by a $40 \% \mathrm{HF}$ bath.

\section{Preparation and characterization of the Parylene film}

Reference silicon chips (diced from a new silicon wafer, $10 \mathrm{~mm}$ by $10 \mathrm{~mm}$ in size) were put both inside and outside the deposition chamber to measure the thickness of the deposited Parylene $\mathrm{C}$ films. The deposition experiments were conducted in the commercialized Parylene deposition machine (PDS 2010 special coating system). After the deposition, the reference silicon chips inside the 
a

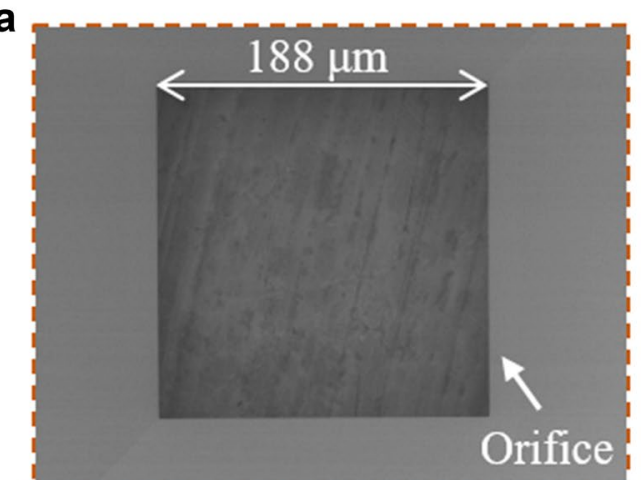

Sealed

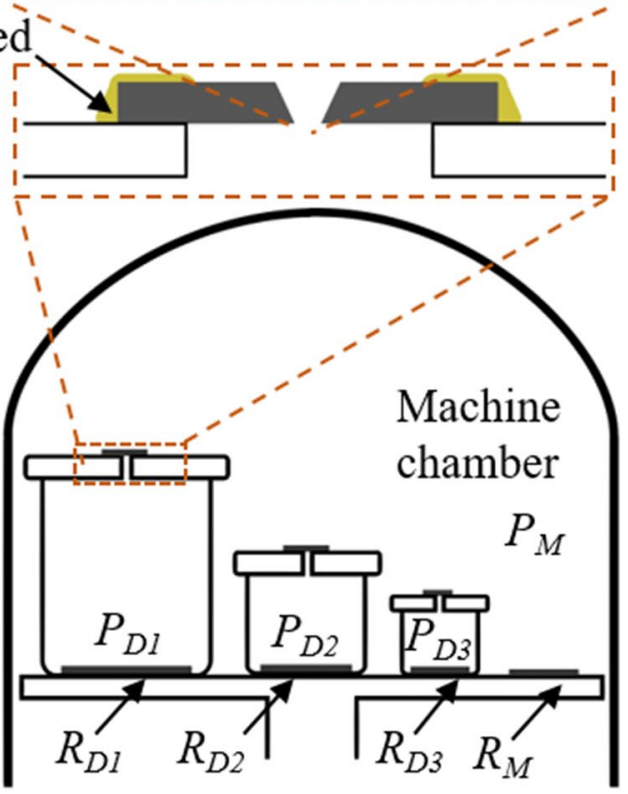

b

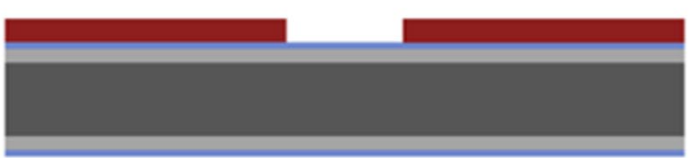

Deposition and patterning of $\mathrm{Si}_{3} \mathrm{~N}_{4} / \mathrm{SiO}_{2}$
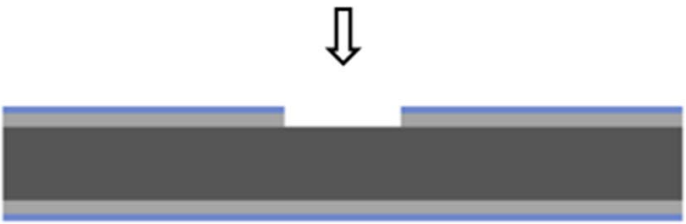

Dry etching of $\mathrm{Si}_{3} \mathrm{~N}_{4} / \mathrm{SiO}_{2}$ with RIE<smiles>[CH]=C</smiles>

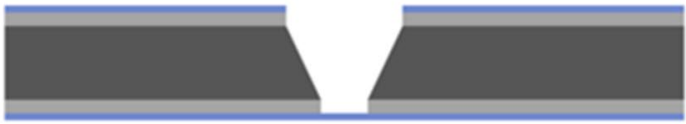

Wet etching of Si by $\mathrm{KOH}$ bath

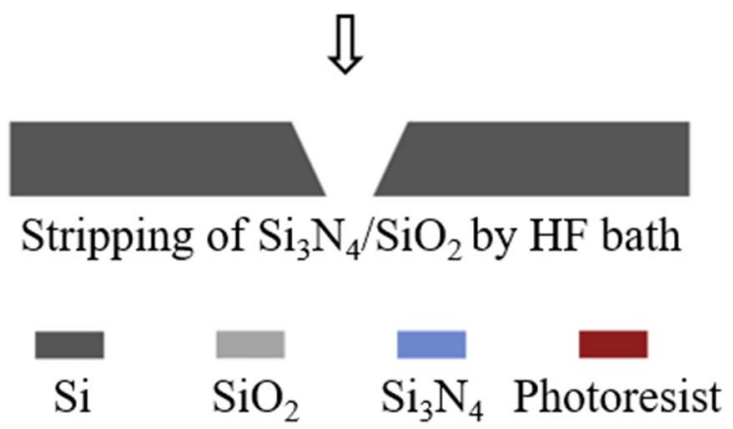

Fig. 2 Working principle of the present ultrathin Parylene deposition (effusion-based deposition). a The schematic of deposition system with a typical SEM image of the microfabricated orifice. $\mathbf{b}$ Schematic of the fabrication process of the orifice chip

deposition chamber, where an ultra-thin Parylene C film was deposited, were scanned by AFM (Bruker, dimension icon) for the thickness, after partially scarping off, and the surface roughness measurements. Surface roughness of directly deposited Parylene film was also measured for comparison. Thickness uniformity was verified by testing the thickness at 5 points on a 4-inch silicon wafer.

\section{Free molecular flow model}

The relationships between the deposition rate $\left(R_{M}\right.$ and $R_{D}$ ) and the monomer pressure are assumed to be the same inside and outside the deposition chamber, which can be written as,

$$
\begin{aligned}
& R_{M}=R_{d} P_{M} \\
& R_{D}=R_{d} P_{D}
\end{aligned}
$$

where $R_{d}$ is a parameter related to temperature, sticking coefficient and molecular weight according to Ref. [12]. The subscripts $M$ and $D$ denote the parameters inside the machine chamber and the deposition chamber, respectively. The pressure here refers partial pressure of the Parylene monomer.

As aforementioned, the effusion process of Parylene monomer through the orifice is in the free molecular flow regime. In the condition when the pressure in both the machine chamber and the deposition chamber are stable, the Parylene monomer flux, $Q$, that passes through the orifice can be expressed as [13],

$$
Q=\frac{1}{4} v A\left(P_{M}-P_{D}\right)
$$


where $v$ is the thermal velocity of Parylene monomers, i.e. $\sqrt{2 k_{B} T / N}$, where $k_{B}$ is the Boltzman constant, $T$ is the temperature, and $N$ is the mass of Parylene $\mathrm{C}$ monomer. $A$ is the opening area of orifice.

In steady state, all the Parylene monomers that enter the deposition chamber will be deposited onto the inner surface of the chamber, including the wafers or samples inside, i.e. no monomer can flee out of the deposition chamber. Therefore, the relationship between the deposition rate inside the deposition chamber and the gas flux can be written as,

$$
Q=k_{B} T \frac{d n}{d t}=k_{B} T \frac{\rho S_{D} R_{D}}{N}
$$

where $n$ is the number of Parylene monomer deposited onto the inner surface of the deposition chamber, $\rho$ is density of the Parylene $C, S_{D}$ is the sticking coefficient.

By solving Eqs. (1) to (4), we derive the ratio of deposition rate between the direct deposition (outside the deposition chamber, $R_{M}$ ) and the present effusion-based ultra-thin deposition (inside the deposition chamber, $R_{D}$ ),

$$
\frac{R_{M}}{R_{D}}=1+4 k_{B} T \frac{\rho R_{d} S_{D}}{v A N}
$$

For the square-shaped orifice,

$$
A=d^{2}
$$

where $d$ stands for the side length of the orifice. The Kn is

$$
\mathrm{Kn}=\frac{\lambda}{d}
$$

where $\lambda$ stands for the mean free path of gas, i.e. $k_{B} T /\left(\sqrt{2} \pi x^{2} P_{M}\right)$, where $x$ is the molecular diameter of Parylene monomer. Therefore, by substituting Eqs. (6) and (7) into Eq. (5), the relationship between the ratio of $R_{M}$ to $R_{D}$ and $\mathrm{Kn}$ can be written as,

$$
\frac{R_{M}}{R_{D}}=1+4 k_{B} T \frac{R_{d} S_{D}}{v N \lambda^{2}} \mathrm{~K}_{\mathrm{n}}^{2}=1+\frac{4 \sqrt{2} \pi^{2} R_{d} \rho S_{D} x^{4} P_{M}^{2}}{N^{\frac{1}{2}}\left(k_{B} T^{\frac{3}{2}}\right)} \mathrm{Kn}^{2}
$$

\section{Numerical simulation of pressure distribution}

The pressure distribution inside the deposition chamber $\left(P_{D}\right)$ was numerically obtained by COMSOL Multiphysics with a free molecular flow model to verify the uniformity of deposition inside the chamber. The boundary condition of the chamber surface was set as $J_{i}=\left(1-f_{i}\right) G_{i}$, where $J_{i}$ represents the bouncing-back molecular flux, $G_{i}$ represents the incoming molecular flux, and $f_{i}$ represents the sticking coefficient, the fraction of the molecules that deposited onto the surface among those that hit the surface. The boundary condition of the orifice was set as a reservoir where the pressure was constantly equal to the partial pressure of the Parylene monomer in the machine chamber $\left(P_{M}\right)$. A parameter sweep of the orifice size was conducted to study the influence of the orifice size.

\section{Results and discussion Deposition output}

The deposition outputs at varied orifice sizes and deposition chamber sizes were shown in Fig. 3a. The deposition output as low as $0.08 \mathrm{~nm} / \mathrm{g}$ was successfully obtained with the orifice diameter of $90 \mu \mathrm{m}$ and the chamber area of $43,332 \mathrm{~mm}^{2}$, although not forming a continuous film, verified under AFM. The chamber area means the internal surface area of the custom-made deposition chamber
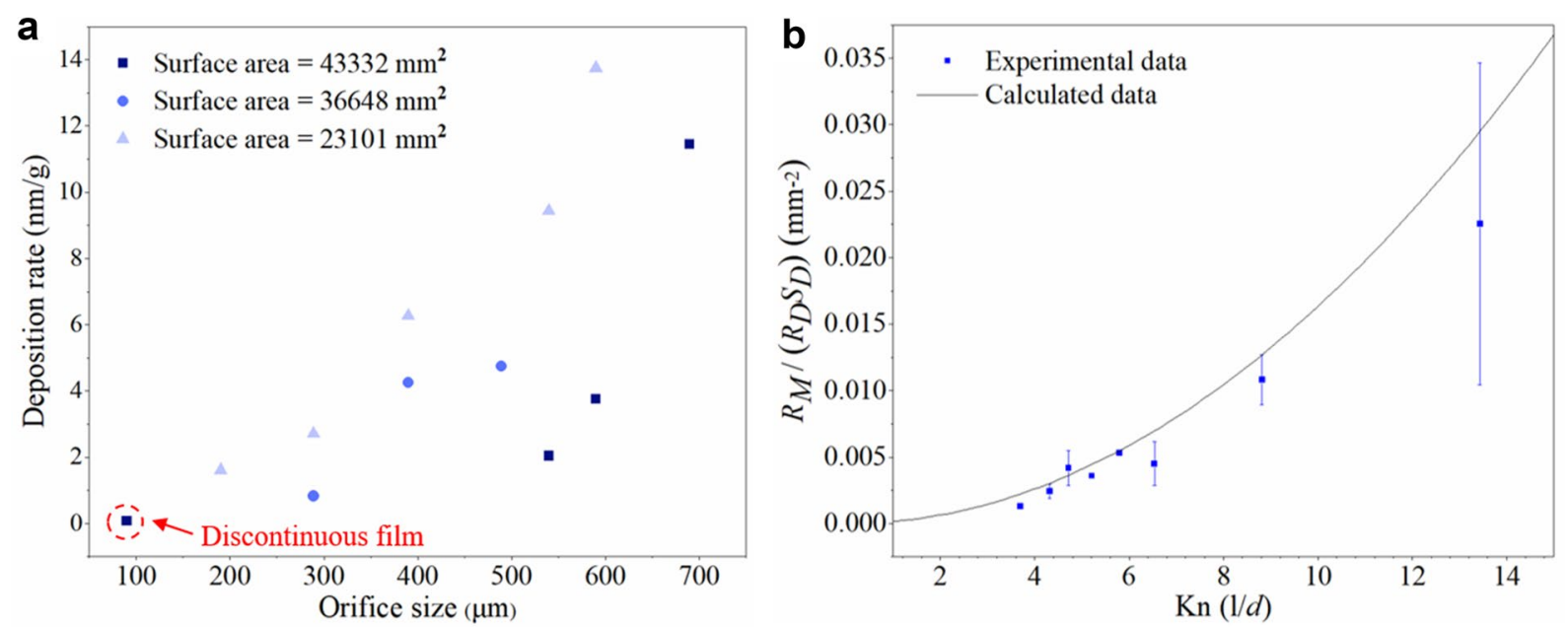

Fig. 3 Deposition outputs. a The deposition rates in different chambers with different orifice sizes, and $\mathbf{b}$ comparison of the experimental results and the model, calculated by Eq. (7) 
unless otherwise indicated. It could be even lowered by using a smaller-sized orifice and larger-areaed chamber, while in the present work (thickness obtained in the machine chamber was around $10 \mu \mathrm{m}$ ), it would be hard to measure the thickness of the deposited film, as it is too low. Even though, the deposition output is $1 / 20,000$ of the conventional deposition in PDS 2010 (following the recipe in the authors' lab). Therefore, this technique fulfilled a highly controllable and reliable thin Parylene deposition.

The ratio of deposition rate between direct deposition (outside the deposition chamber) and effusion-based deposition (inside the deposition chamber) against $\mathrm{Kn}$ is shown in Fig. 3, along with the calculated prediction of the model. Comparing with the established model, i.e. Eq. (7), as shown in Fig. 3b, the present thin Parylene deposition can be well described by the free molecular flow model. Thus, the present ultrathin Parylene deposition is predictable and designable, which allowed a reliable ultrathin Parylene preparation and can be used to precisely tune the surface properties of the deposition substrate.

\section{The distribution of Parylene monomer in the chamber}

Uniformity of the thin Parylene film deposited inside the chamber is critical for real applications. Although Parylene monomers entered the deposition chamber just through a small orifice, thanks to the relatively small of the free molecular path inside the chamber and the ultra-low sticking coefficient of Parylene deposition, the distribution of the Parylene monomer inside the deposition chamber is highly uniform, as shown in Fig. 4a, which guaranteed a uniform Parylene deposition. Experiment results also proved a uniform Parylene film were successfully obtained by the present work. Typical thickness variation at 4-inch wafer scale was $0.14 \mathrm{~nm}$ (91.65 $\mathrm{nm}$ thick). This further confirmed the present thin Parylene deposition technique is highly controllable and reliable. By using orifices with different diameters, multiple samples with different Parylene thicknesses can be easily prepared in a single deposition run. As a preliminary demonstration, 4 4-inch wafers with different thicknesses of $91.65 \pm 0.14 \mathrm{~nm}$ (I, orifice diameter of $690 \mu \mathrm{m}$ ), $40.78 \pm 3.34 \mathrm{~nm}$ (II, $640 \mu \mathrm{m}$ ), $19.43 \pm 0.91 \mathrm{~nm}$ (III, $590 \mu \mathrm{m})$, and $15.58 \pm 2.11 \mathrm{~nm}(\mathrm{IV}, 540 \mu \mathrm{m})$ were successfully obtained in one run.

\section{Surface roughness characterization}

The surface roughness of thin Parylene film prepared by the direct deposition and the present effusion-based deposition were compared in Fig. 5. As shown in Fig. 5a, the surface roughness of the Parylene film prepared by the present method were larger than those prepared by the direct deposition. Meanwhile, the power spectral density, as shown in Fig. 5b, further indicated the Parylene film prepared by the conventional dimer mass determined deposition had smaller and uniform rough units. This may come from the case that at an ultra-low pressure, the

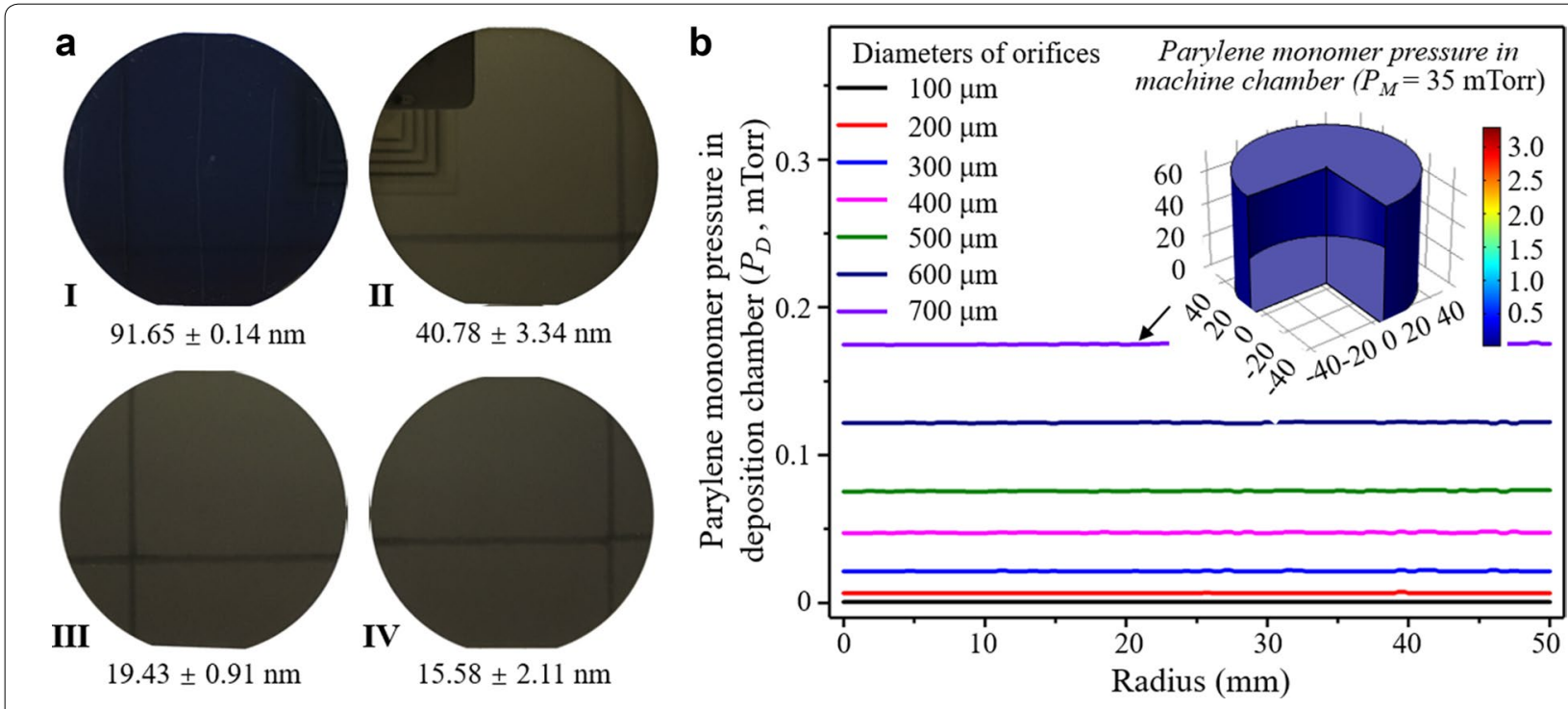

Fig. 4 Uniformity of the present effusion-based ultra-thin Parylene deposition. a Photos of the so-deposited ultra-thin Parylene films on 4-inch silicon wafers. The values of thicknesses were shown on bottom of the wafers. The patterns on the wafer II, III, and IV with different colors were the shadows of the ceiling in the clean room and did not actually exist on the wafers. $\mathbf{b}$ Numerical result of the monomer pressure distributions in the deposition chambers of different sized orifices. The inset shows a typical monomer pressure distribution inside the deposition chamber 

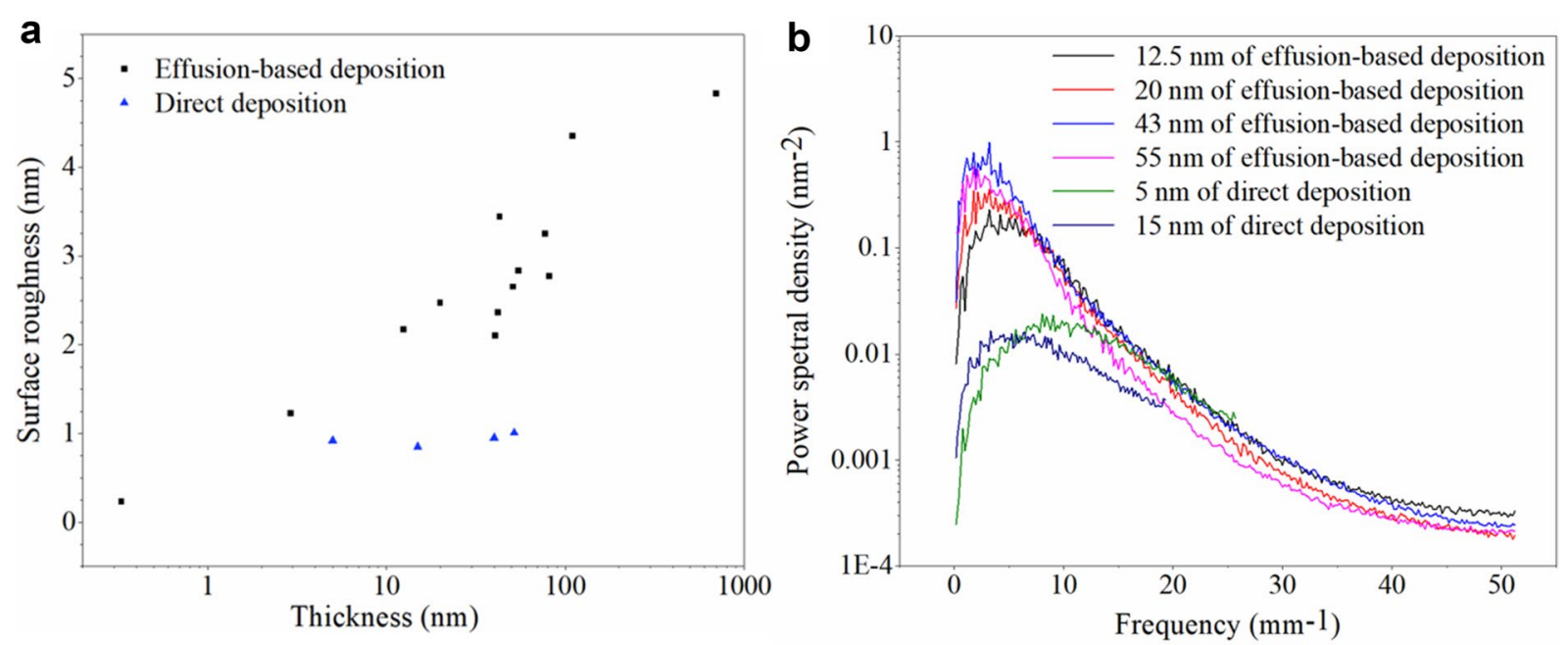

Fig. 5 Surface roughness of the direct deposition and the present effusion-based ultra-thin Parylene deposition measured by AFM surface scanning (a) and according power spectral density (b)

case of our ultra-thin Parylene deposition, the film deposition prefers an island mode, which thereby led to a relatively rough surface.

\section{Conclusions}

By using a precisely defined effusion orifice connecting the home-made deposition chamber and the machine chamber, an ultra-low Parylene deposition output, down to $0.08 \mathrm{~nm} / \mathrm{g}$ (with the orifice diameter of $90 \mu \mathrm{m}$ and the chamber area of $43,332 \mathrm{~mm}^{2}$ ), and typical thickness variation at 4-inch wafer scale was $0.14 \mathrm{~nm}(91.65 \mathrm{~nm}$ thick) was successfully achieved, which enable a highly controllable and reliable ultra-thin Parylene deposition. The technique will find promising applications in flexible electronics or biomedical microdevices, especially for those who required precise surface functionalization or passivation.

\section{Authors' contributions}

YL and WW drafted the manuscript. YL, WW and YT conceived of the study. HL and DK worked on the model calculation. YL and WD carried out the deposition experiment. All authors read and approved the final manuscript.

\section{Author details}

${ }_{1}^{1}$ Institute of Microelectronics, Peking University, Beijing 100871, China

${ }^{2}$ Electrical Engineering, Caltech, Pasadena, CA 91125, USA. ${ }^{3}$ National Key Lab of Micro/Nano Fabrication Technology, Beijing 100871, China.

\section{Acknowledgements}

The authors thank Prof. Ying Yan at Dalian University of Technology for her help in analysis of the AFM measurements.

\section{Competing interests}

The authors declare that they have no competing interests.
Ethics approval and consent to participate Not applicable.

\section{Funding}

Funding for this research was provided by and thank the financial supports from the scholarship from the Major State Basic Research Development Program (973 Program) (Grant No. 2015CB352100) and the National Natural Science Foundation of China (Grant Nos. 81471750 and 91323304).

\section{Publisher's Note}

Springer Nature remains neutral with regard to jurisdictional claims in published maps and institutional affiliations.

Received: 30 August 2018 Accepted: 8 October 2018

Published online: 10 October 2018

\section{References}

1. Wang RX, Huang XJ, Liu GF, Wang W, Dong FT, Li ZH (2010) Fabrication and characterization of a Parylene-based three-dimensional microelectrode array for use in retinal prosthesis. J Microelectromech Syst 19(2):367-374

2. Yu HQ, Zheng NG, Wang W, Wang S, Zheng XX, Li ZH (2013) Electroplated nickel multielectrode microprobes with flexible Parylene cable for neural recording and stimulation. J Microelectromech Syst 22(5):1199-1206

3. Yu HQ, Xiong WJ, Zhang HX, Wang W, Li ZH (2014) A Parylene self-locking cuff electrode for peripheral nerve stimulation and recording. J Microelectromech Syst 23(5):1025-1035

4. Lei YH, Liu YP, Wang W, Wu WG, Li ZH (2011) Studies on Parylene C-caulked PDMS (pcPDMS) for low permeability required microfluidics applications. Lab Chip 11:1385-1388

5. Liu YP, Zhang LQ, Mo C, Cao YP, Wu WG, Wang W (2016) Caulking polydimethylsiloxane molecular networks by thermal chemical vapor deposition of Parylene C. Lab Chip 16:4220-4229

6. Wang W, Kang DY, Tai YC (2016) Reliable deposition of ultra-thin parylene. In: Proceeding of the 29th IEEE international conference on micro electro mechanical systems (IEEE MEMS' 2016), pp 462-464

7. Nishinaka Y, Jun R, Prihandana GS, Miki N (2013) Fabrication of polymer microneedle electrodes coated with nanoporous Parylene. Jpn J Appl Phys 52:06GL10 
8. Rapp BE, Boigt A, Dirschka M, Lange K (2012) Deposition of ultrathin Parylene $C$ films in the range of $18 \mathrm{~nm}$ to $142 \mathrm{~nm}$ : controlling the layer thickness and assessing the closeness of the deposited films. Thin Solid Films 520(15):4884-4888

9. Von Metzen RP, Lass N, Ruther P, Stieglitz T (2011) Diffusion-limited deposition of Parylene C. J Microelectromech Syst 20(1):239-251

10. Lin M, Chen QY, Wang ZW, Fang YC, Liu JF, Yang YC, Wang W, Cai YM, Huang R (2017) Flexible polymer device based on Parylene-C with memory and temperature sensing functionalities. Polymer 9(8):310
11. Kang DY, Matsuki S, Tai YC (2015) Study of the hybrid parylene/PDMS material. In: Proceeding of the 28th IEEE international conference on micro electro mechanical systems (IEEE MEMS' 2015), pp 397-400

12. Fortin JB, LU TM (2002) A model for the chemical vapor deposition of Poly(para-xylylene) (Parylene) thin films. Chem Mater 14(5):1945-1949

13. Ivchenko IN, Loyalka SK, Tompson RV (eds) (2007) The free-molecular regime. In: Analytical methods for problems of molecular transport. Fluid mechanics and its applications, vol 83. Springer, Dordrecht, pp 91-140

\section{Submit your manuscript to a SpringerOpen ${ }^{\circ}$ journal and benefit from:}

- Convenient online submission

- Rigorous peer review

- Open access: articles freely available online

- High visibility within the field

- Retaining the copyright to your article

Submit your next manuscript at $\boldsymbol{\nabla}$ springeropen.com 